\title{
Synergistic Interface Layer Optimization and Surface Passivation with Fluorocarbon Molecules toward Efficient and Stable Inverted Planar Perovskite Solar Cells
}

\author{
Long Zhou, ${ }^{1}$ Jie Su, ${ }^{1}$ Zhenhua Lin, ${ }^{1}$ Xing Guo, ${ }^{1}$ Jing Ma, ${ }^{1}$ Tao Li, ${ }^{2}$ Jincheng Zhang, ${ }^{1,3}$ \\ Jingjing Chang $\mathbb{D}^{1,3}$ and Yue Hao ${ }^{1,3}$ \\ ${ }^{1}$ State Key Discipline Laboratory of Wide Band Gap Semiconductor Technology, Shaanxi Joint Key Laboratory of Graphene, \\ School of Microelectronics, Xidian University, 2 South Taibai Road, Xi'an 710071, China \\ ${ }^{2}$ Centre for Spintronics and Quantum System, State Key Laboratory for Mechanical Behavior of Materials, School of Materials Science \\ and Engineering, Xi'an Jiaotong University, Xi'an, Shaanxi 710049, China \\ ${ }^{3}$ Advanced Interdisciplinary Research Center for Flexible Electronics, Xidian University, 2 South Taibai Road, Xi'an 710071, China
}

Correspondence should be addressed to Jingjing Chang; jjingchang@xidian.edu.cn

Received 18 November 2020; Accepted 9 June 2021; Published 28 June 2021

Copyright (C) 2021 Long Zhou et al. Exclusive Licensee Science and Technology Review Publishing House. Distributed under a Creative Commons Attribution License (CC BY 4.0).

\begin{abstract}
Large-size organic halide passivation has been considered an efficient approach to enhance the perovskite solar cell (PSC) efficiency and stability. Herein, a facile posttreatment strategy was demonstrated, wherein trifluoromethyl-phenethylamine hydrobromide $\left(\mathrm{CF}_{3}-\mathrm{PEABr}\right)$ is firstly used to passivate the perovskite film surface. The $\mathrm{CF}_{3}-\mathrm{PEABr}$ surface posttreatment could coordinate with halide dangling bonds that exist at the perovskite crystal surface. Moreover, the surface treatment with $\mathrm{CF}_{3}-\mathrm{PEABr}$ could efficiently passivate the defects in the perovskite film and suppress the nonradiative carrier recombination. As a result, a high efficiency of $21.3 \%$ is obtained, and an increment of $80 \mathrm{mV}$ in $V_{\text {oc }}$ (a large $V_{\text {oc }}$ of $1.15 \mathrm{~V}$, with a $0.42 \mathrm{~V}$ voltage deficit) occurs, compared to the control device. To relieve the hydrophobic nature properties of the $-\mathrm{CF}_{3}$ functional group and the dewetting problem of PCBM layer deposition, a surfactant Triton X-100 is used to modify the PCBM layer. Furthermore, the devices with $\mathrm{CF}_{3}$-PEABr posttreatment exhibit better operational, thermal $\left(85^{\circ} \mathrm{C}\right)$, and long storage stabilities without any encapsulation.
\end{abstract}

\section{Introduction}

The organic-inorganic hybrid perovskite has received increasing attention owing to its unique and remarkable optoelectronic properties [1-8]. The certified power conversion efficiency (PCE) of single-junction solar cells based on perovskite thin films has reached $25.5 \%$ until now [9]. Thus, the perovskite solar cell (PSC) has been heralded as a newgeneration photovoltaic technology owing to its low-cost and solution-processed fabrication [10-13]. Despite such brilliant outcomes, both device efficiency and stability are found to be dramatically degenerated by the defects existing in amorphous regions, film surfaces, and grain boundaries [14-17]. To reduce defects in perovskite films and further improve the device efficiency and stability, miscellaneous strategies were exploited, such as composition engineering, additive engineering, interface layer engineering, and surface passivation engineering [18-22]. Meanwhile, massive energy loss $\left(E_{\text {loss }}\right)$ observed in perovskite solar cells could impair the ultimate efficiency, which suggests that the nonradiative recombination is induced by the trap-state density and defects in perovskite films $[23,24]$.

Up to now, long-chain cations or large-radius organic cations are widely used in surface passivation engineering, which could further enhance the device open-circuit voltage $\left(V_{\mathrm{oc}}\right)$ and fill factor (FF) by suppressing the defects in the perovskite film [25-29]. Recently, Zheng et al. demonstrated a trace amount of surface-anchoring alkylamine ligands to modify the interface and grain, which could enhance the charge carrier mobility and reduce the trap-state density of perovskite films [30]. They achieved a certified stabilized PCE of $22.3 \%$ with an excellent $V_{\text {oc }}$ of $1.17 \mathrm{~V}$ and remarkable operating stability with over $1000 \mathrm{~h}$ under continuous illumination. Yang et al. designed a new passivation molecule, 
wherein D-4-tert-butylphenylalanine was used to passivate perovskite defects by combining all effective passivation groups [31]. They reported a remarkable small $V_{\text {oc }}$ deficit of $0.34 \mathrm{~V}$ and a high efficiency of $21.6 \%$ with a large $V_{\text {oc }}$ of $1.23 \mathrm{~V}$. Furthermore, Gharibzadeh et al. reported a significant improvement via mixed $2 \mathrm{D} / 3 \mathrm{D}$ perovskites to obtain an efficiency of $19.4 \%$ and a large $V_{\text {oc }}$ of $1.31 \mathrm{~V}$ [32]. Additionally, Jiang's research group used a large organic salt phenethylammonium iodide (PEAI) to modify the perovskite film surface for defect passivation, and a certified efficiency of $23.32 \%$ with a large $V_{\text {oc }}$ of $1.18 \mathrm{~V}$ was obtained, which approximated to the Shockley-Queisser limit [14]. Zhou et al. firstly demonstrated that by introducing 2-(4-fluorophenyl)ethyl ammonium iodide and a fluorinated aromatic cation to form $2 \mathrm{D} / 3 \mathrm{D}$ perovskites as the absorbing layer, the photovoltaic devices achieved a high $V_{\text {oc }}$ of $1.12 \mathrm{~V}$, leading to a stabilized efficiency of $20.54 \%$, and the device maintained $99 \%$ of the initial efficiency after $864 \mathrm{~h}$ [33]. Thereafter, Zhou et al. investigated the fluorination position (ortho-, meso-, and para-) effect on the aromatic moiety and obtained a PCE of $20.1 \%$ with a remarkable $V_{\text {oc }}$ of $1.21 \mathrm{~V}$ [34]. Recently, Zhu et al. used fluorinated BAI to treat the perovskite film and introduced 4-(trifluoromethyl)pyridine (TFP) as an additive to increase the HTL's hydrophobicity, which could suppress the nonradiative charge carrier recombination and enhance the PSC's resistance to moisture and device stability [35]. As viewed by these results, the search for alternative hydrophobic large cations for perovskite passivation to achieve high performance is still a challenge. As a result, it is highly significant to achieve larger $V_{\mathrm{oc}}$ and high efficiency for further commercial applications of the optoelectronic device.

Herein, we demonstrated a novel approach to modify the perovskite surface via depositing phenethylamine hydrobromide with the trifluoromethyl functional group $\left(-\mathrm{CF}_{3}\right)$ and ammonium group to suppress the trap-state density and improve the photovoltaic performance and moisture stability. A large organic salt containing positively charged cations $\left(\mathrm{CF}_{3}-\mathrm{PEA}^{+}\right)$could coordinate with halide dangling bonds that exist at the crystal surface and passivate defects arising from vacancies of halide ions or organic cations on the perovskite film [36, 37]. Meanwhile, we introduced a surfactant (Triton X-100) to modify the PCBM layer, which could provide potential interaction with the perovskite surface due to the hydrophilic polyethylene oxide chain and self-assemble on the surface of the PCBM layer with the hydrophobic group. As a result, the device with the synergistic effect of $\mathrm{CF}_{3}$-PEABr modification and surfactant Triton X-100 demonstrates a high PCE of $21.3 \%$ with a large $V_{\text {oc }}$ of $1.15 \mathrm{~V}$ and an excellent $\mathrm{FF}$ of $81.4 \%$. Moreover, the $\mathrm{CF}_{3}-\mathrm{PEABr}-$ modified device shows better stability than the conventional device.

\section{Results and Discussion}

The perovskite film $\mathrm{MA}_{1-y} \mathrm{FA}_{y} \mathrm{PbI}_{3-x} \mathrm{Cl}_{x}$ was deposited using the two-step spin coating method, which is reported in our previous works $[7,11]$. After perovskite film annealing, the $\mathrm{CF}_{3}$-PEABr/IPA solution ( $2 \mathrm{mg} / \mathrm{mL}$ in IPA) was used to treat the perovskite film surface further, generating a $2 \mathrm{D} / 3 \mathrm{D}$ perovskite heterostructure [7]. We firstly investigated the XRD spectra of perovskite films with and without $\mathrm{CF}_{3}$ PEABr treatment, as shown in Figure 1(a). Both films exhibit obviously intense diffraction peaks located at $14.1^{\circ}, 28.4^{\circ}$, and $31.8^{\circ}$ which correspond to the (110), (220), and (310) typical crystal planes of the tetragonal phase. It is obvious that the peak located at $5.4^{\circ}$ appears in the perovskite film with $\mathrm{CF}_{3}-\mathrm{PEABr}$ passivation, which means the formation of low-dimensional perovskites. To further clarify that lowdimensional perovskite structure, we measured the XRD pattern of $2 \mathrm{D}$ perovskite $\left(\mathrm{CF}_{3}-\mathrm{PEA}\right)_{2} \mathrm{PbI}_{2} \mathrm{Br}_{2}$ prepared by $\mathrm{CF}_{3}-\mathrm{PEABr}$ and $\mathrm{PbI}_{2}$. The low-angle diffraction peak of mixed $2 \mathrm{D} / 3 \mathrm{D}$ perovskite measured is consistent with the peak of $2 \mathrm{D}$ perovskite $\left(\mathrm{CF}_{3}-\mathrm{PEA}\right)_{2} \mathrm{PbI}_{2} \mathrm{Br}_{2}$ (Figure $\mathrm{S} 1$ ). Meanwhile, the $d$-space of low-dimensional perovskites was calculated as $16.38 \AA$ using Bragg's law which is also in good agreement with the $2 \mathrm{D}$ perovskite $\left(\mathrm{CF}_{3}-\right.$ PEA $)_{2} \mathrm{PbI}_{2} \mathrm{Br}_{2}$ 's value (16.59 $\AA$ ) [13]. Furthermore, lowdimensional perovskites could be confirmed by the PL spectrum that a small peak corresponding to lowdimensional perovskites appears at around $525 \mathrm{~nm}$ (Figure S2). Moreover, the peak intensity of perovskite films with $\mathrm{CF}_{3}$-PEABr treatment is slightly higher than that of conventional films, indicating higher film crystallinity with surface passivation. Meanwhile, a slightly narrowed full width at half maximum (FWHM) can be observed for perovskite films with $\mathrm{CF}_{3}-\mathrm{PEABr}$ treatment, which means less defect density, higher crystallinity, and better film quality, as shown in Figure 1(a) and Figure S2. Figure 1(b) depicts the UV-vis absorption spectra of all perovskite films. The optical band edge of conventional perovskite films is located at $790 \mathrm{~nm}$, consisting of a bandgap of $1.57 \mathrm{eV}$. There is a blueshift behavior for the optical band edge of perovskite films with $\mathrm{CF}_{3}-\mathrm{PEABr}$ surface passivation, indicating a larger optical bandgap for the top perovskite thin film (Figure S3). This result is in good agreement with other large cations reported in previous works [29]. Notably, the perovskite film with $\mathrm{CF}_{3}-\mathrm{PEABr}$ treatment exhibits higher absorption intensity, which is profited from its high film quality and surface passivation. Furthermore, the surface morphology of perovskite films was investigated via scanning electron microscopy (SEM) measurement in Figures 1(c) and 1(d). Uniform and dense morphologies are observed for both films. It is obvious that stripy grains are observed in the perovskite film with $\mathrm{CF}_{3}$ $\mathrm{PEABr}$ passivation, which means that the large organic cations $\mathrm{CF}_{3}-\mathrm{PEA}^{+}$caused layer structure formation. Notably, $\mathrm{CF}_{3}-\mathrm{PEABr}$ has an alkyl hydrophobic chain on account of its $-\mathrm{CF}_{3}$ functional group. We further investigated the water contact angles of perovskite films with or without $\mathrm{CF}_{3}-\mathrm{PEABr}$ treatment. By contrast, the perovskite film with $\mathrm{CF}_{3}-\mathrm{PEABr}$ displays a much larger water contact angle $\left(74.70^{\circ}\right)$ than the conventional film $\left(44.98^{\circ}\right)$. The result indicates that the $\mathrm{CF}_{3}-\mathrm{PEABr}$ assembles on the perovskite film surface, which dramatically increases the hydrophobicity of the film. This hydrophobicity could not only potentially increase the film stability but also generate the dewetting problem of PCBM layer deposition. 


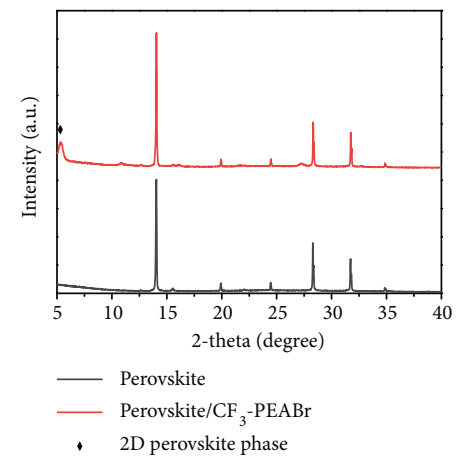

(a)

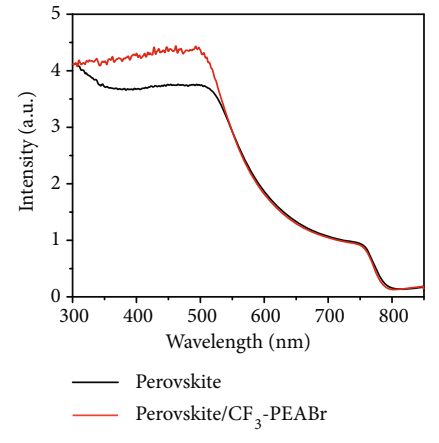

(b)

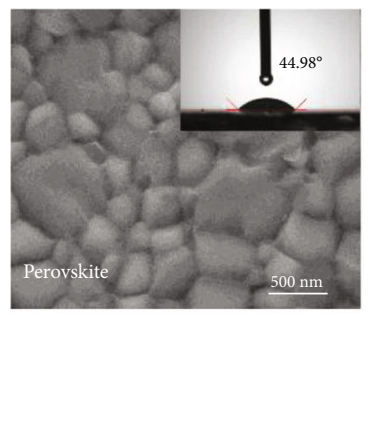

(c)

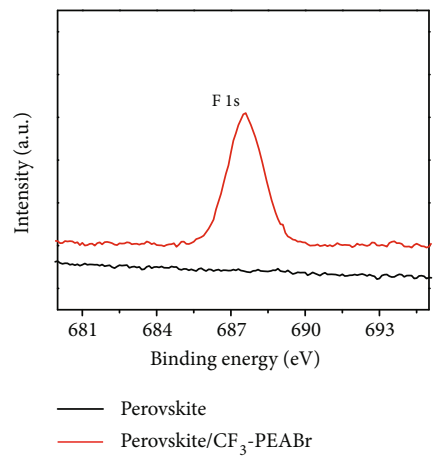

(f)

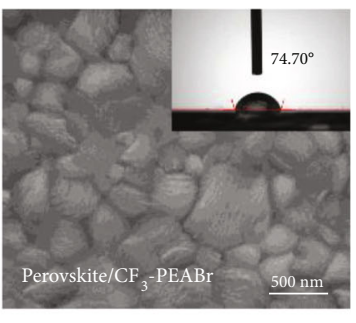

(d)

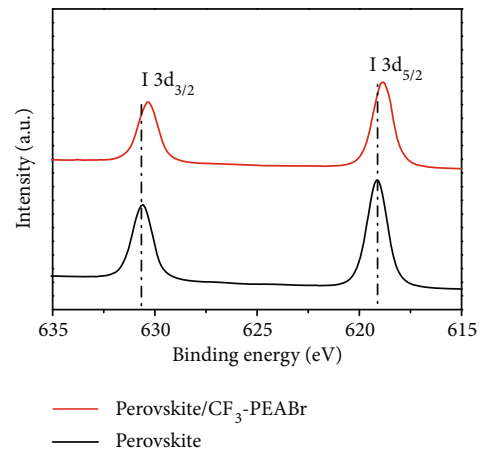

(g)

Figure 1: Perovskite material characterization and passivation schematic. (a) $\mathrm{XRD}$ spectra of conventional and $\mathrm{CF}_{3}-\mathrm{PEABr}-\mathrm{treated}$ perovskite films. (b) Absorption spectra of conventional and $\mathrm{CF}_{3}-\mathrm{PEABr}$-treated perovskite films. (c, d) SEM images of conventional and $\mathrm{CF}_{3}$-PEABr-treated perovskite films. Inset shows the water contact angle images. (e) Schematic model of the perovskite solar cell with $\mathrm{CF}_{3}-\mathrm{PEABr}$ passivation. (f, $\mathrm{g}$ ) XPS curves of perovskite films with or without $\mathrm{CF}_{3}-\mathrm{PEABr}$ passivation.

Furthermore, we investigated the roughness of the perovskite film surface via atomic force microscopy (AFM) measurement, as shown in Figure S4. The perovskite film with $\mathrm{CF}_{3}-\mathrm{PEABr}$ passivation exhibits smaller roughness $(11.8 \mathrm{~nm})$ than the conventional film $(15.4 \mathrm{~nm})$, which is advantageous for upper layer deposition and interface quality. As shown in Figure 1(e), the unavoidable loss of halide ions or organic cations on the surface of conventional perovskite films leads to defects (including halide interstitial, halide vacancy, and $\mathrm{Pb}$ vacancy) because of the small formation energy [38]. In principle, $\mathrm{CF}_{3}-\mathrm{PEABr}$ contains an ammonium group and a trifluoromethyl group. The ammonium group could form hydrogen bonding with halide ions grown on the surface of perovskites due to stronger electrostatic interactions. As a result, the $\mathrm{CF}_{3}$ $\mathrm{PEABr}$ can suppress the formation of halide dangling bonds, vacancies, and defects located at grain boundaries [39, 40]. On the other hand, the ultrathin low-dimensional perovskite formed on the $3 \mathrm{D}$ perovskite surface due to the induced $\mathrm{CF}_{3}-\mathrm{PEA}^{+}$ions and the hydrophobic trifluoromethyl functional group $-\mathrm{CF}_{3}$ exists at the terminal of the crystal, acting as the barrier to prevent the $\mathrm{H}_{2} \mathrm{O}$ from ambient air into perovskite films in high-humidity environments. Similar results have been verified by X-ray photoelectron spectroscopy (XPS), as shown in Figures 1(f) and 1(g). It is obvious that the emerged $\mathrm{F} 1 \mathrm{~s}$ peaks (at $687.6 \mathrm{eV}$ ) and $\mathrm{C}-\mathrm{F}$ bond are mainly attributed to the incorporation of $\mathrm{CF}_{3}$ -
$\mathrm{PEA}^{+}$cations for the film with surface passivation, indicating the presence of $\mathrm{CF}_{3}-\mathrm{PEABr}$ on the film surface. Furthermore, the smaller binding energy of the I peak is discovered after $\mathrm{CF}_{3}-\mathrm{PEABr}$ passivation, which mainly results from the strong positively charged $\mathrm{NH}_{3}{ }^{+}$end of the $\mathrm{CF}_{3}$ - $\mathrm{PEABr}$ molecule. The $\mathrm{NH}_{3}{ }^{+}$end of the $\mathrm{CF}_{3}-\mathrm{PEABr}$ molecule could grow on the surface of perovskite films and form a hydrogen bond with halide ions, which weakens the interaction between $\mathrm{Pb}$ and I [36]. Due to the electrondonating nature of the Lewis base, the $\mathrm{Pb}$ and I peaks of the film with $\mathrm{CF}_{3}-\mathrm{PEABr}$ treatment shifted toward lower binding energy, which further verifies the interaction between surface defects such as the uncoordinated $\mathrm{Pb}^{2+}$ or halide dangling bond and $\mathrm{CF}_{3}-\mathrm{PEABr}$ molecule. Meanwhile, the $\mathrm{C}=\mathrm{O}$ peak located at $288.1 \mathrm{eV}$ is observed and mainly induced by the decomposition under ambient conditions during measurement (Figure S6), while the intensity of the $\mathrm{C}=\mathrm{O}$ peak obviously decreases after $\mathrm{CF}_{3}-\mathrm{PEABr}$ passivation, corroborating the better moisture stability due to the hydrophobic trifluoromethyl functional group. Moreover, the modification of $\mathrm{CF}_{3}$-PEABr is further confirmed by the $\mathrm{N}$ 1s region in X-ray photoelectron spectroscopy (XPS). The $\mathrm{N}$ 1s spectrum reveals that the typical bond of the $\mathrm{C}-\mathrm{NH}_{2}$ group is induced by $\mathrm{FA}^{+}$cations or $\mathrm{CF}_{3}-\mathrm{PEA}^{+}$cations and the bond of the $\mathrm{C}=\mathrm{NH}_{2}^{+}$group is induced by $\mathrm{FA}^{+}$cations in the perovskite film. Compared with the control perovskite film, the perovskite/ $\mathrm{CF}_{3}-\mathrm{PEABr}$ film exhibits a higher ratio 

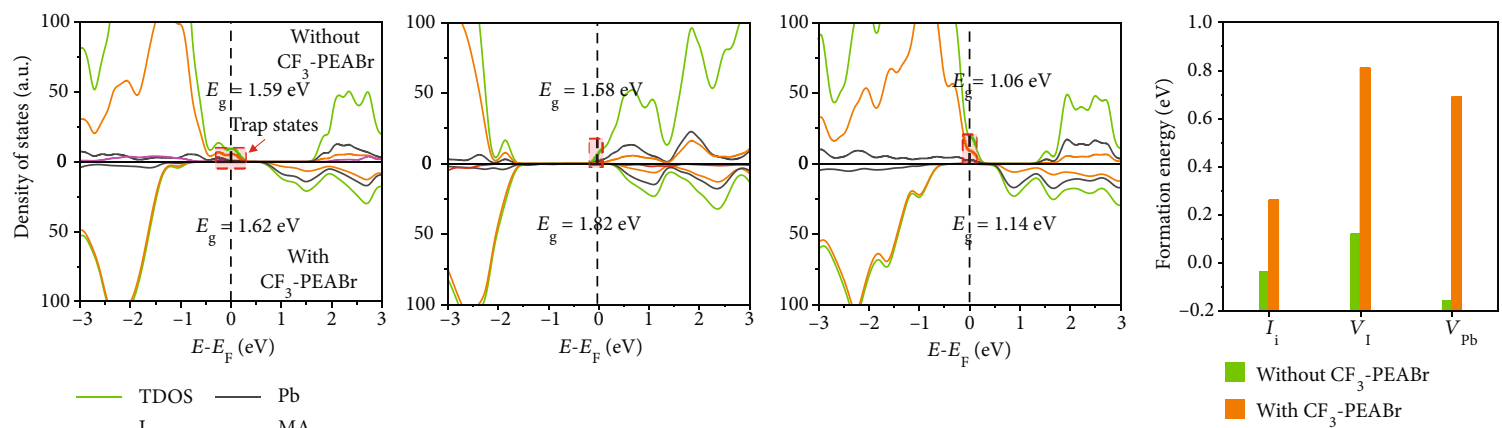

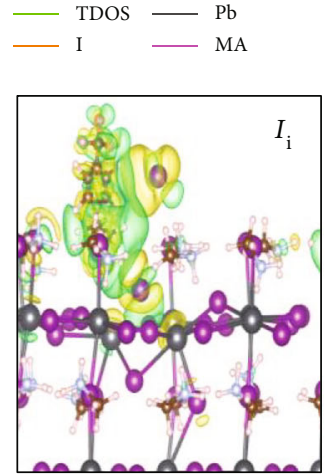

(a)

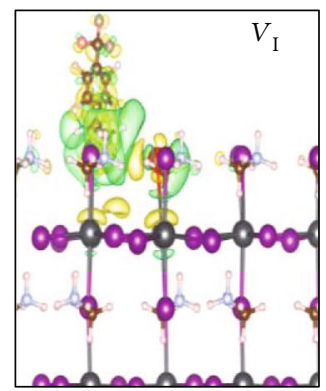

(b)

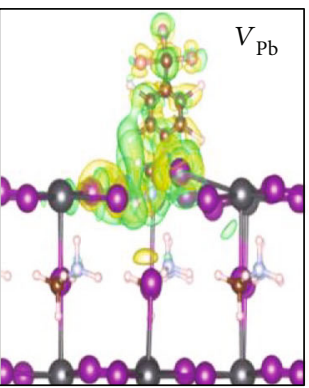

(c) (d)

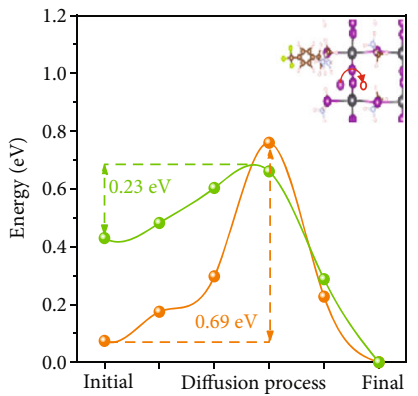

(e)

Figure 2: DFT calculation for $\mathrm{CF}_{3}-\mathrm{PEABr}$ passivation. (a-c) Density of states and charge density difference of the $\mathrm{CF}_{3}-\mathrm{PEABr}-\mathrm{passivated}$ MAI surface with (a) $I_{\mathrm{i}}$ and (b) $V_{\mathrm{I}}$ and the (c) PbI surface with $V_{\mathrm{Pb}}$. (d) Formation energies of $I_{\mathrm{i}}, V_{\mathrm{I}}$, and $V_{\mathrm{Pb}}$ at the perovskite surface. (e) The diffusion energy curves of iodide ion in the MAI surface with and without $\mathrm{CF}_{3}-\mathrm{PEABr}$ passivation, where the migration energy is marked by the arrows. The inserts are the diffusion paths in the perovskite.

of the $\mathrm{C}-\mathrm{NH}_{2}$ group which is mainly attributed to the introduction of $\mathrm{CF}_{3}-\mathrm{PEABr}$. Furthermore, it is obvious that the peaks of the perovskite/ $\mathrm{CF}_{3}-\mathrm{PEABr}$ film shift toward lower binding energy, which further suggests that $\mathrm{CF}_{3^{-}}$ $\mathrm{PEABr}$ has a strong molecular interaction with the perovskite surface. Fourier transform infrared spectroscopy (FTIR) measurements are used to confirm the possible chemical interaction in perovskite films. The strengthening and broadened profile of the $\mathrm{N}-\mathrm{H}$ bending vibration band $\left(1550-1600 \mathrm{~cm}^{-1}\right)$ could further demonstrate the molecular interaction in the perovskite/ $\mathrm{CF}_{3}-\mathrm{PEABr}$ film compared with the control film (Figure S5) [13, 41, 42].

To reveal the passivation mechanism of $\mathrm{CF}_{3}-\mathrm{PEABr}$, we calculated the density of states (DOS) and charge transfer of $\mathrm{MAPbI}_{3}$ absorbed with $\mathrm{CF}_{3}-\mathrm{PEABr}$ by density functional theory (DFT). Both the I interstitial $\left(I_{\mathrm{i}}\right)$ and I vacancy $\left(V_{\mathrm{I}}\right)$ at the MAI-terminated surface and the $\mathrm{Pb}$ vacancy $\left(V_{\mathrm{Pb}}\right)$ at the PbI-terminated surface were considered. As shown in Figure 2(a), the $I_{\mathrm{i}}$ introduces a defect state in the bandgap (about $1.59 \mathrm{eV}$ ) and serves as a hole trap. The $\mathrm{CF}_{3}-\mathrm{PEABr}$ could supply electrons to the perovskite and passivate these hole trap states, suggesting reduced nonradiative recombination for $\mathrm{CF}_{3}$-PEABr-passivated perovskite. Meanwhile, such electron transfer could tune the position of the Fermi level related to the band edges of perovskite and then improve the band offset. As a result, the bandgap of perovskite is slightly enlarged by the $\mathrm{CF}_{3}-\mathrm{PEABr}$, which is consistent with the above experimental analysis. A similar electron transfer and enlarged bandgap are also observed for $\mathrm{CF}_{3}-\mathrm{PEABr}$ that passivated the $V_{\mathrm{I}}$ and $V_{\mathrm{Pb}}$. Meanwhile, no defect states are formed in the bandgap upon absorbing the $\mathrm{CF}_{3}-\mathrm{PEABr}$, as exhibited in Figures 2(b) and 2(c). In addition, it could be observed from Figure 2(d) that the formation energy of these defects increases after incorporation of $\mathrm{CF}_{3}-\mathrm{PEABr}$ since the $\mathrm{Br}$ anion could occupy the $V_{\mathrm{I}}$, and the $\mathrm{H}$ of the ammonium group could bond with the iodine dangling bond (as displayed in Figures 2(a)-2(c)). These bonds significantly reduce the initial energy of the I ion migration process and then enlarge the migration energy of I ion and suppress I ion migration, as displayed in Figure 2(e). As a result, the $\mathrm{CF}_{3}$-PEABr preferentially bounds to the perovskite surface and stabilizes the perovskite surface structure.

To investigate the carrier dynamics in perovskite films with the effect of $\mathrm{CF}_{3}-\mathrm{PEABr}$ passivation, we carried out the steady-state photoluminescence (PL) measurement and time-resolved PL (TRPL) decay measurements, as shown in Figures 3(a) and 3(b). The carrier lifetime can be extracted with a double exponential decay model [42], as listed in Table S1. After $\mathrm{CF}_{3}-\mathrm{PEABr}$ surface passivation, the increased peak intensity and blueshift behavior are observed, indicating efficient surface passivation and lower trap-state density in perovskite films. Meanwhile, the perovskite film with $\mathrm{CF}_{3}-\mathrm{PEABr}$ passivation possesses a longer carrier lifetime (269.1 ns) than the conventional film (127.4 ns), indicating that nonradiative carrier recombination is effectively suppressed. In addition, we have checked the effect of surface passivation on the band alignment via ultraviolet photoelectron spectroscopy (UPS) measurement, 


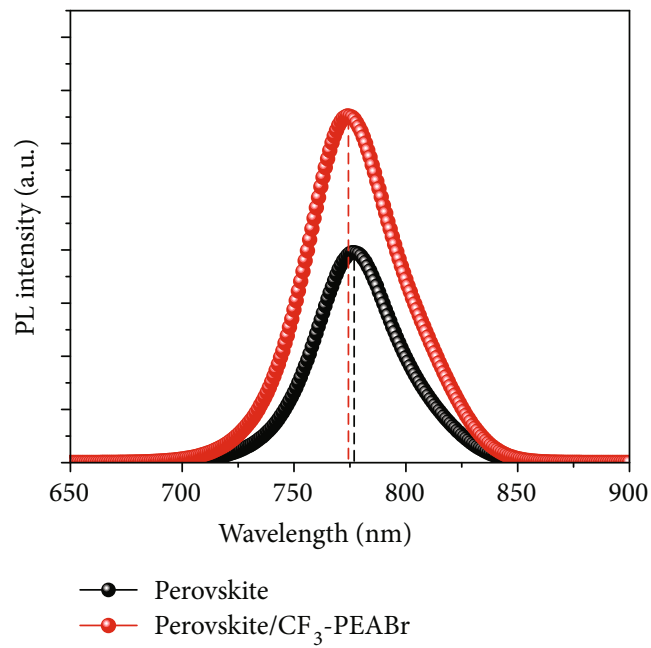

(a)

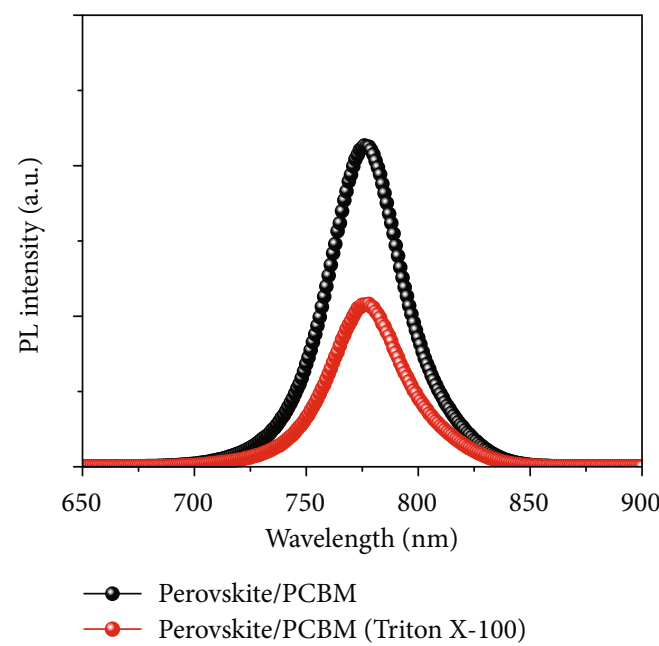

(c)

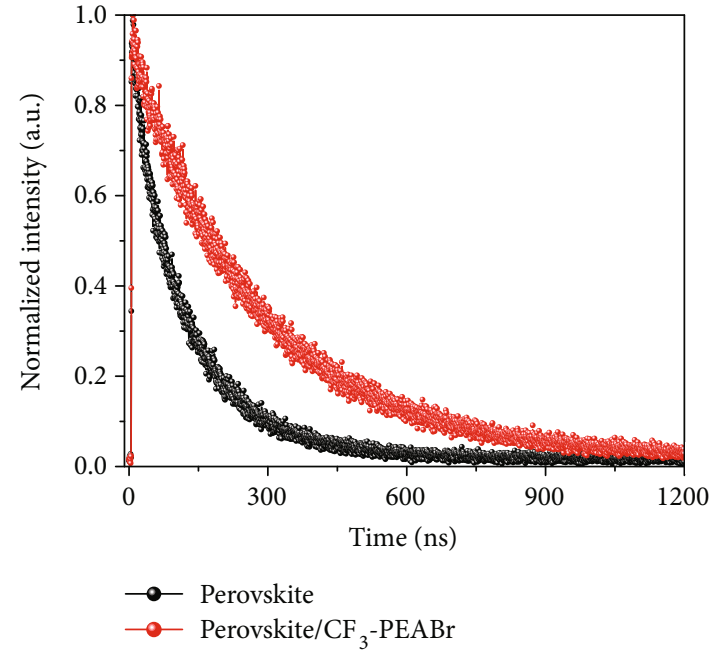

(b)

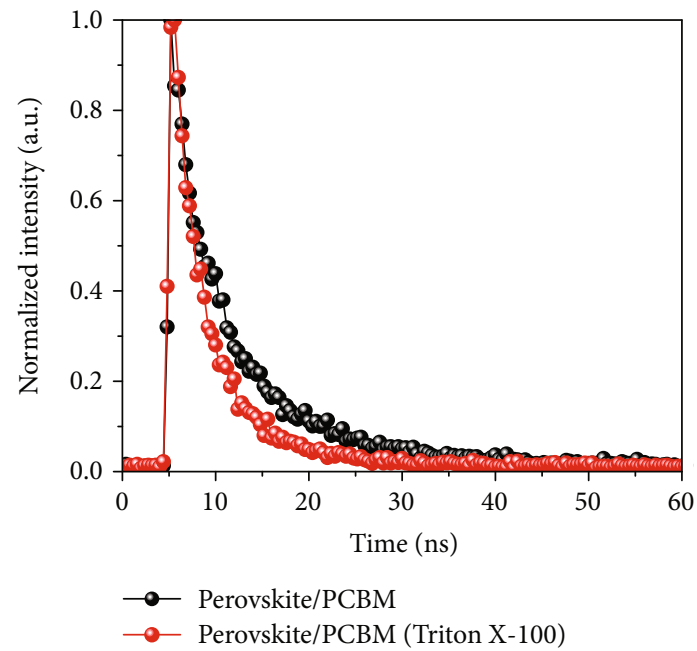

(d)

FIGURe 3: Carrier dynamics for perovskite films. (a) PL and (b) TRPL spectra of perovskite films with or without $\mathrm{CF}_{3}-\mathrm{PEABr}$ treatment. (c) PL and (d) TRPL spectra of perovskite films with PCBM or PCBM (Triton X-100) layers.

as shown in Figure S7. We found that the perovskites with $\mathrm{CF}_{3}-\mathrm{PEABr}$ passivation showed a smaller work function value and better band alignment between the perovskite and the PCBM layer, which indicated an efficient charge transfer process between the perovskite and the PCBM layer. Furthermore, work functions were further checked with surface potential via Kelvin probe force microscopy (KPFM), as shown in Figure S8. A higher surface potential for the perovskite film with $\mathrm{CF}_{3}-\mathrm{PEABr}$ treatment was discovered, indicating the decreased work function with the $\mathrm{CF}_{3}-\mathrm{PEABr}$ modification. The apparent decrease in work functions of perovskites with $\mathrm{CF}_{3}-\mathrm{PEABr}$ modification could form a band bending between the perovskite and the PCBM layer, which could reconfigure the interfacial energy band structure, leading to enhanced built-in potential and charge collection.

The poor coverage of the PCBM layer on the hydrophobic perovskite film is another challenge preventing excellent device performance. As discussed above, the perovskite film with $\mathrm{CF}_{3}-\mathrm{PEABr}$ treatment has evident hydrophobic proper- ties owing to the $-\mathrm{CF}_{3}$ functional group, arousing the problem that the PCBM layer cannot be successfully spin-coated on the perovskite film. For the $\mathrm{CF}_{3}-\mathrm{PEABr}$ surface passivation, the devices with a pure PCBM layer exhibited a terrible PCE (Figure S9) due to the poor PCBM coverage caused by the hydrophobic surface of the perovskite film. Thus, it is necessary to use a surfactant to relieve the nonwetting problem of the perovskite film. Triton X-100 is one of the nonionic surfactants and is widely used in biochemical and industrial processes due to its amphiphilic structure. The Triton X-100 molecule includes a hydrophilic polyethylene oxide chain and an aromatic hydrocarbon group, as shown in Figure S10. Thus, here, we introduced a surfactant (Triton X-100) with a hydrophilic polyethylene oxide chain to modify the PCBM layer. And Triton X-100 exhibits a terminal hydroxyl group, which could provide potential interaction such as hydrogen bonding or electrostatic interaction with the $\mathrm{CF}_{3}$ of the perovskite film surface, which can promote the successful PCBM deposition on the 


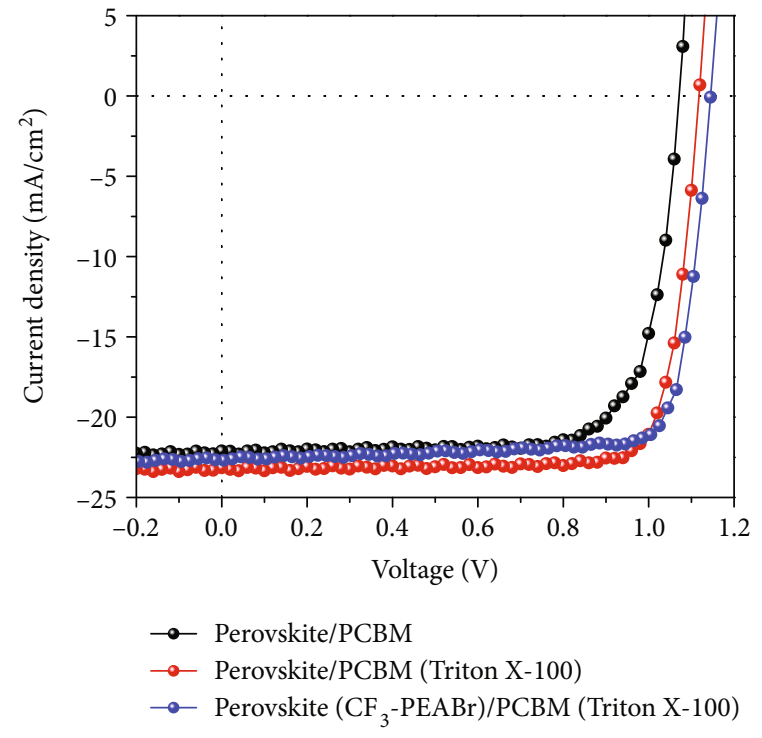

(a)

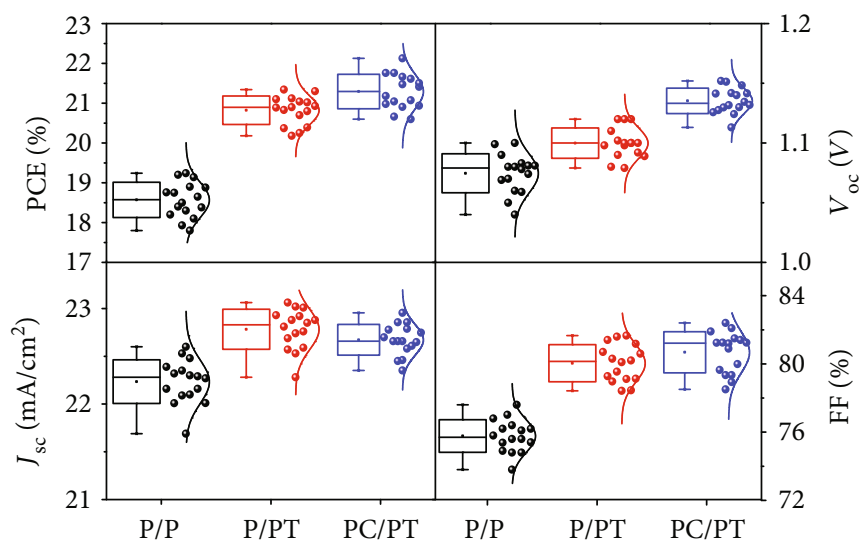

(c)

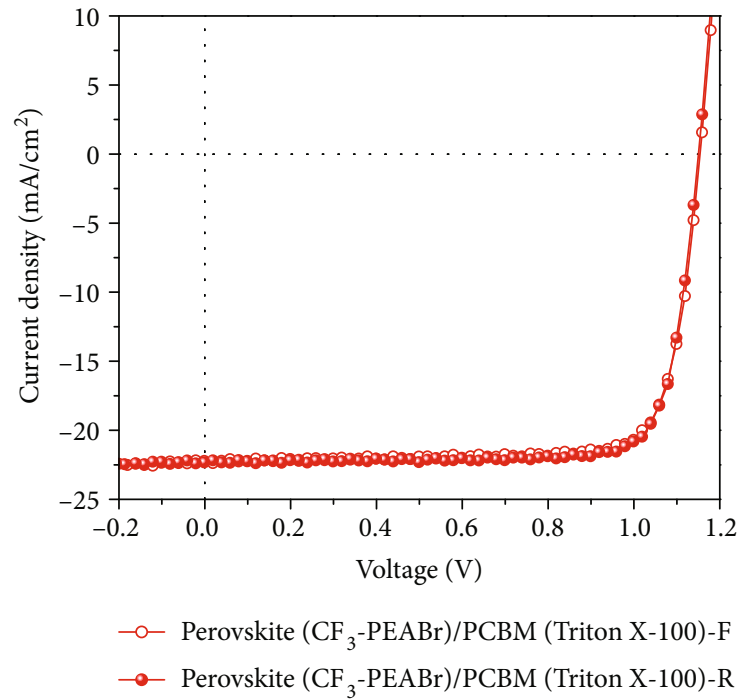

(b)

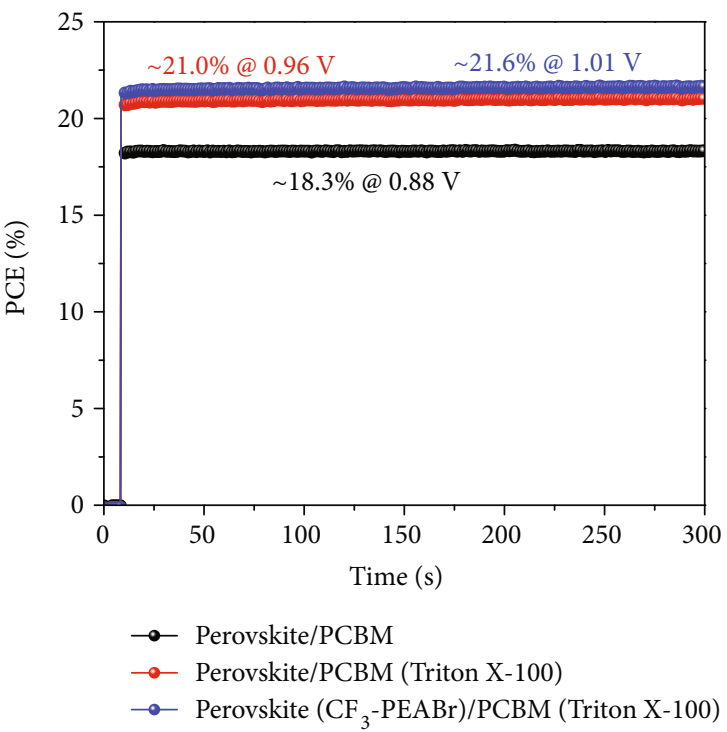

(d)

FIGURE 4: Device performance of PSCs for different conditions. (a) $J$ - $V$ curves of perovskite devices with different conditions. (b) Hysteresis behaviors of perovskite devices based on perovskite $\left(\mathrm{CF}_{3}-\mathrm{PEABr}\right) / \mathrm{PCBM}$ (Triton $\left.\mathrm{X}-100\right)$. (c) Distribution of device performance with different conditions. P/P: perovskite/PCBM; P/PT: perovskite/PCBM (Triton X-100); PC/PT: perovskite $\left(\mathrm{CF}_{3}-\mathrm{PEABr}\right) / \mathrm{PCBM}(\mathrm{Triton}$ $\mathrm{X}-100)$. (d) Steady-state output of current density and PCE at the maximum power point.

perovskite film with $\mathrm{CF}_{3}-\mathrm{PEABr}$ passivation. Furthermore, we investigated the carrier transport kinetics between the perovskite and the PCBM layer, as shown in Figures 3(c) and $3(\mathrm{~d})$. By contrast, obvious PL quenching behavior is observed for the perovskite/PCBM (Triton X-100), which indicates the efficient carrier transport from the perovskite to the PCBM layer. Additionally, the lifetime of the sample with PCBM (Triton X-100) (4.30 ns) is smaller than that of the sample with PCBM (7.24 ns), which validates that the surfactant (Triton X-100) could improve the interfacial contact between $\mathrm{PCBM}$ and $\mathrm{CF}_{3} \mathrm{PEABr}$, which enhances the electron extraction and transport from the perovskite to the PCBM layer (Table S2).
In order to provide insight into the effect of the $\mathrm{CF}_{3}$ $\mathrm{PEABr}$ surface passivation, the inverted planar $\mathrm{p}$-i-n-type PSC devices were fabricated. The architecture of the solar cell based on the $\mathrm{NiO}_{x}$ hole transport layer is presented in Figure 1(e). Figure 4(a) shows the current density-voltage $(J-V)$ curves of devices, and Table 1 lists the detailed parameters of device performance. The control device exhibits an average PCE of $18.5 \%$, a $V_{\text {oc }}$ of $1.07 \mathrm{~V}$, a $J_{\text {sc }}$ of $22.2 \mathrm{~mA} / \mathrm{cm}^{2}$, and an $\mathrm{FF}$ of $76.5 \%$. In contrast, the device with synergistic passivation of $\mathrm{CF}_{3}-\mathrm{PEABr}$ treatment and surfactant (Triton X-100) modification shows an increased PCE of $21.3 \%$ with a large $V_{\text {oc }}$ of $1.15 \mathrm{~V}$, a $J_{\mathrm{sc}}$ of $22.7 \mathrm{~mA} / \mathrm{cm}^{2}$, and an $\mathrm{FF}$ of $81.4 \%$. The champion device exhibits a high PCE of $21.9 \%$. 
TABLE 1: Average device performance parameters for perovskite solar cells with different modifications. The average PCE data were calculated from at least 36 devices.

\begin{tabular}{lcccccc}
\hline Condition & $V_{\mathrm{oc}}(\mathrm{V})$ & $J_{\mathrm{sc}}\left(\mathrm{mA} / \mathrm{cm}^{2}\right)$ & $\mathrm{FF}(\%)$ & PCE (\%) (best device) & $R_{\mathrm{s}}\left(\Omega \mathrm{cm}^{2}\right)$ & $R_{\mathrm{sh}}\left(\mathrm{k} \Omega \mathrm{cm}^{2}\right)$ \\
\hline Perovskite/PCBM & $1.07 \pm 0.04$ & $22.2 \pm 0.6$ & $76.5 \pm 3.2$ & $18.5(19.6)$ & 3.13 & 1.51 \\
Perovskite/PCBM (Triton X-100) & $1.11 \pm 0.02$ & $23.3 \pm 0.5$ & $80.7 \pm 2.5$ & $21.0(21.5)$ & 2.68 & 3.45 \\
Perovskite (CF 3 -PEABr)/PCBM (Triton X-100) & $1.15 \pm 0.02$ & $22.7 \pm 0.3$ & $81.4 \pm 2.8$ & $21.3(21.9)$ & 2.94 & 2.78 \\
\hline
\end{tabular}

The slightly improved current density mainly results from the ultrathin low-dimensional perovskite formed on the 3D perovskite surface, and excess amounts of large cations absorbed on the perovskite crystal surface could physically limit carrier transport and lead to the decreased photocurrent [43], which has been verified in the above results. The device has a significant improvement in PCE than the control device due to the great enhancement in $V_{\mathrm{oc}}$ and $\mathrm{FF}$, which may result from the higher-quality perovskite film, lower trap density, and better band alignment (Figure S7). In comparison, the device with PEABr surface passivation also fabricated and exhibited a PCE of $20.6 \%$ with a $V_{\text {oc }}$ of $1.12 \mathrm{~V}$, but this $V_{\text {oc }}$ is still smaller than that of the device with $\mathrm{CF}_{3}-\mathrm{PEABr}$ passivation because of the electronwithdrawing $-\mathrm{CF}_{3}$ group introduction (Figure $\mathrm{S} 11$ ). $\mathrm{CF}_{3}-$ $\mathrm{PEABr}$ could provide hydrogen bonding between $\mathrm{H}$ of the ammonium group and halide ions or electrostatic interactions which can be credited to the electronwithdrawing $\mathrm{F}$ atom. As a result, it can avoid the formation of halide dangling bonds and vacancy-related defects and efficiently improve film quality. In order to check the effect of surfactant Triton X-100, we fabricated the device with a modified PCBM (Triton X-100) layer based on the conventional perovskite film. Unexpectedly, it showed a significantly improved PCE of $21.0 \%$ as a consequence of the increased $J_{\text {sc }}$ of $23.3 \mathrm{~mA} / \mathrm{cm}^{2}, V_{\text {oc }}$ of $1.11 \mathrm{~V}$, and FF of $80.7 \%$, compared with the conventional device. It can be concluded that the $J_{\mathrm{sc}}$ improvement is mainly caused by Triton X-100 modification, while the $V_{\text {oc }}$ improvement is mainly caused by $\mathrm{CF}_{3}$-PEABr surface passivation, and the synergistic effect could be achieved to obtain the best device performance. All samples with modification of $\mathrm{CF}_{3}-\mathrm{PEABr}$ or Triton X-100 have been carefully optimized with different concentrations to achieve the best performance (Figures S12 and S13). Additionally, negligible hysteresis behavior appeared in both the forward and reverse scan directions for all samples, as shown in Figure 4(b) and Figure S14. In addition, the stronger electrostatic interactions and hydrogen bonding coordination via $\mathrm{CF}_{3}-$ $\mathrm{PEABr}$ posttreatment upon the surface can block the accumulation of charge at the interface. Meanwhile, Figure 4(c) shows the device performance distribution of PSCs under different conditions, indicating good reliability and uniformity for the device fabrication. To further verify device $J_{\mathrm{sc}}$, the external quantum efficiency (EQE) spectra and the integrated $J_{s c}$ are depicted in Figure S15. The device with a modified PCBM (Triton X-100) layer based on the conventional perovskite film and $\mathrm{CF}_{3}-\mathrm{PEABr}$ passivation has a larger integrated $J_{\mathrm{sc}}$ value $\left(22.8 \mathrm{~mA} / \mathrm{cm}^{2}\right.$ and $22.3 \mathrm{~mA} / \mathrm{cm}^{2}$ ), which is much higher than that of the conventional device $\left(21.9 \mathrm{~mA} / \mathrm{cm}^{2}\right)$. All of these results are in good agreement with the results extracted from measured $J-V$ curves. Moreover, we have measured the steady-state output of PCE values at the maximum power point (Figure 4(d)). The devices exhibit a steady-state PCE of $18.3 \%, 21.0 \%$, and $21.6 \%$ under different conditions, respectively. All of them show the excellent reliability of the $J-V$ scans and the stability of device performance. Notably, the photocurrent is stabilized within seconds when the light is turned on, which supports the hysteresis-free behavior of the devices.

In addition, we provide insight into the carrier transport and recombination mechanism via the transient photocurrent (TPC) and transient photovoltage (TPV) measurements. Both of them are used to explain the larger $J_{\mathrm{sc}}$ caused by the effect of surfactant Triton X-100, as shown in Figures 5(a) and 5(b). The photocurrent decay lifetime is related to the charge extraction and transport processes, and the photovoltage decay lifetime mainly indicates the charge recombination processes. Noteworthily, the device with surfactant Triton X-100 exhibits faster decay behavior $(0.81 \mu \mathrm{s})$ than the conventional device $(1.32 \mu \mathrm{s})$, indicating more efficient charge extraction and transport near the perovskite surface, which conformed well with EQE results. Figure 5(b) reveals that the photovoltage lifetime of the device with surfactant Triton X-100 $(437 \mu \mathrm{s})$ is larger than that of the conventional device $(228 \mu \mathrm{s})$, indicating the suppressed charge recombination near the perovskite surface. The better carrier transport and suppressed charge recombination are benefited from the interaction between Triton X-100 and perovskite. All of these results are in good agreement with the increased $J_{\mathrm{sc}}$ for Triton X-100-modified PCBM-based devices.

In order to further check the effect of $\mathrm{CF}_{3}-\mathrm{PEABr}$ passivation and surfactant Triton X-100 on carrier extraction and transport mechanisms, the relationship of $V_{o c}$ as a function of light intensity was investigated, as shown in Figure 5(c). The $V_{o c}$ is measured with respect to the light intensity at various light intensities, from 100 to $0.1 \mathrm{~mW} / \mathrm{cm}^{2}$. There is a linear relationship with a slope of $K_{\mathrm{B}} T / q$ between $V_{\mathrm{oc}}$ and light intensity on a semilogarithmic scale, where $K_{\mathrm{B}}$ is the Boltzmann constant, $T$ is the absolute temperature, and $q$ is the elementary charge, respectively. The slope extracted from the curves of the device with $\mathrm{CF}_{3}-\mathrm{PEABr}$ passivation $\left(1.32 K_{\mathrm{B}} T / q\right)$ is much smaller than that of the conventional device $\left(1.86 K_{\mathrm{B}} T / q\right)$, which suggests that $\mathrm{CF}_{3}-\mathrm{PEABr}$ surface passivation results in the minimized energy loss and suppresses carrier recombination with strong coordination. To further confirm the charge transport and recombination 


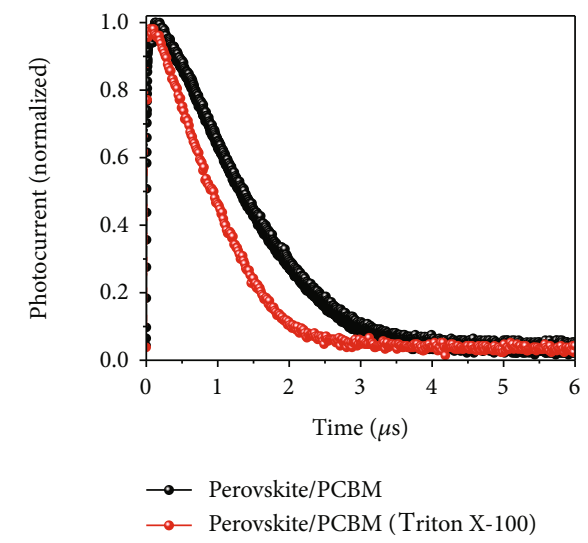

(a)

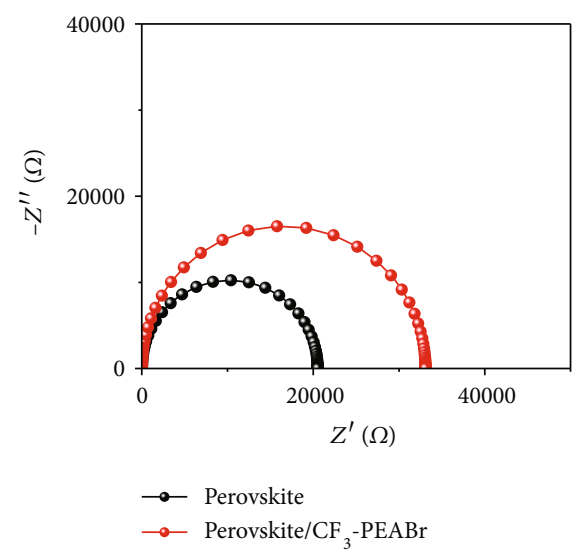

(d)

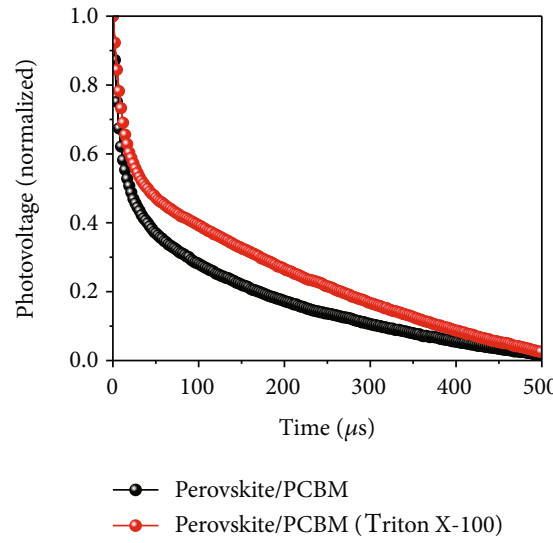

(b)

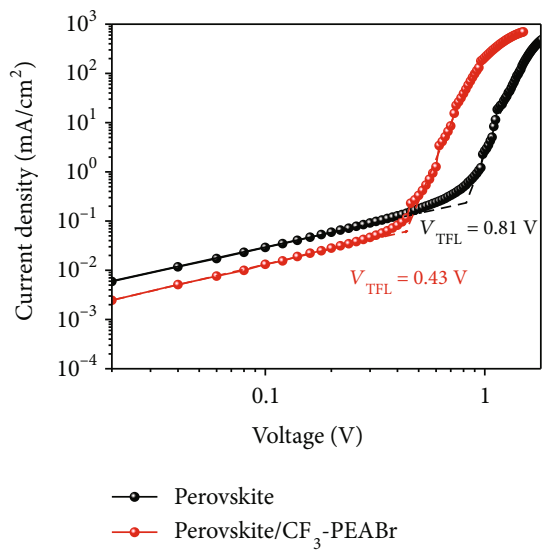

(e)

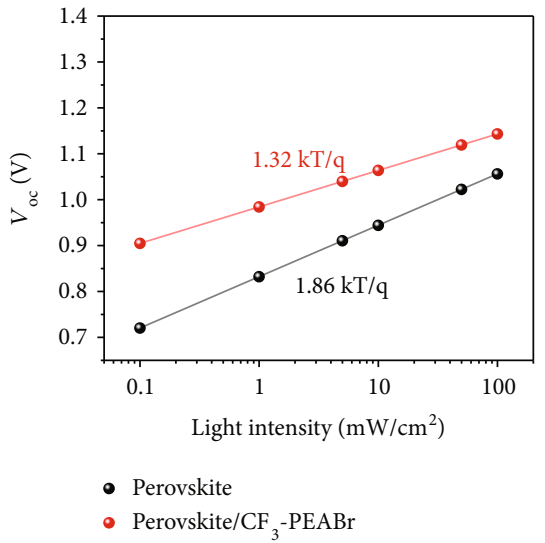

(c)

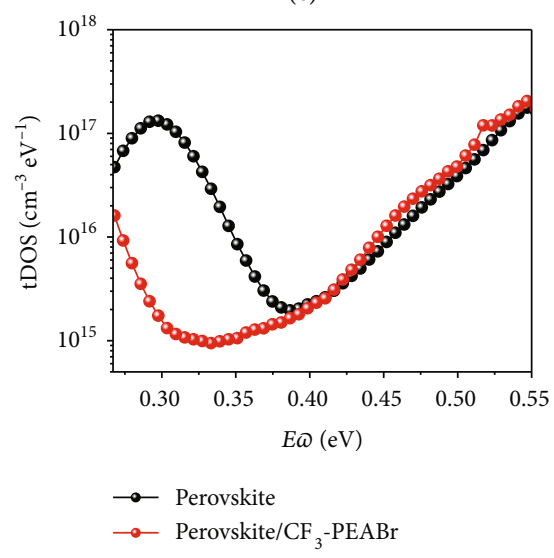

(f)

FIgURE 5: Device-level characterization for different conditions. (a) Transient photocurrent and (b) transient photovoltage measurements of the conventional device and the device with Triton X-100. (c) $V_{\mathrm{oc}}$ as a function of light intensity. (d) EIS of the devices with or without $\mathrm{CF}_{3}$ $\mathrm{PEABr}$ treatment. (e) $I-V$ curves for electron-only devices. (f) tDOS curves of perovskite solar cells with or without $\mathrm{CF}_{3}-\mathrm{PEABr}$ passivation.

process, the electrical impedance spectroscopy (EIS) measurement was carried out to extract the recombination resistance $\left(R_{\text {rec }}\right)$ and series resistance $\left(R_{s}\right)$. The devices are measured in the dark condition with an applied voltage of $1 \mathrm{~V}$, and the corresponding Nyquist is plotted in Figure 5(d). Meanwhile, the fitting parameters extracted with the equivalent circuit are listed in Table S3. The device with $\mathrm{CF}_{3}-\mathrm{PEABr}$ passivation exhibits larger $R_{\text {rec }}$ than the conventional device, indicating the suppressed carrier recombination. This result is well consistent with the $R_{\mathrm{sh}}$ results extracted from $J-V$ curves and the larger $V_{\text {oc }}$ of the device with $\mathrm{CF}_{3}-\mathrm{PEABr}$ passivation. Furthermore, the capacitance-voltage $(C-V)$ characteristics of devices were operated to check the built-in potential $\left(V_{\mathrm{bi}}\right)$, which could be extracted with the Mott-Schottky method [44], as shown in Figure S16. The measurable enhanced $V_{\mathrm{bi}}(1.13 \mathrm{~V})$ compared with that of the conventional device $(0.95 \mathrm{~V})$ means a promoted driving force for photogenerated carrier separation as well as an efficiently suppressed electron-hole recombination. In order to further verify the trap-state density of perovskite films, electron-only devices (ITO/ $\mathrm{SnO}_{2} /$ perovskite/PCBM/Ag) were fabricated. Thus, the trap-filled limit voltage $\left(V_{\mathrm{TFL}}\right)$ is extracted from $I-V$ curves and the trap-state density $\left(n_{\text {trap }}\right)$ is determined from the trap-filled limit voltage according to the equation $V_{\mathrm{TFL}}=$ $e n_{\text {trap }} L^{2} / 2 \varepsilon_{0} \varepsilon$, where $e$ is the electron charge, $L$ is the thickness of the electron-only device, $\varepsilon$ is the relative dielectric constant, and $\varepsilon_{0}$ is the vacuum permittivity $[45,46]$, as shown in Figure 5(e). It is noticed that a smaller $V_{\mathrm{TFL}}$ value $(0.43 \mathrm{~V})$ could be obtained for the perovskite film with $\mathrm{CF}_{3}-\mathrm{PEABr}$ passivation compared with the conventional device $(0.81 \mathrm{~V})$, which indicates that the perovskite film with large cation passivation owns significantly reduced electron trap density $\left(0.86 \times 10^{15} \mathrm{~cm}^{-3}\right)$ than the conventional film $\left(1.62 \times 10^{15} \mathrm{~cm}^{-3}\right)$. Furthermore, a reduced trap density in devices with $\mathrm{CF}_{3}-\mathrm{PEABr}$ passivation was measured by thermal admittance spectroscopy, as shown in Figure 5(f). The device with $\mathrm{CF}_{3^{-}}$ PEABr passivation exhibited lower trap density of states (tDOS) in the shallow trap depth region $(0.25$ to $0.35 \mathrm{eV})$, which is attributed to the passivation of surface defects. A lower shallow trap density means that the defects at grain boundaries were efficiently passivated via the effect of $\mathrm{CF}_{3}$ - 


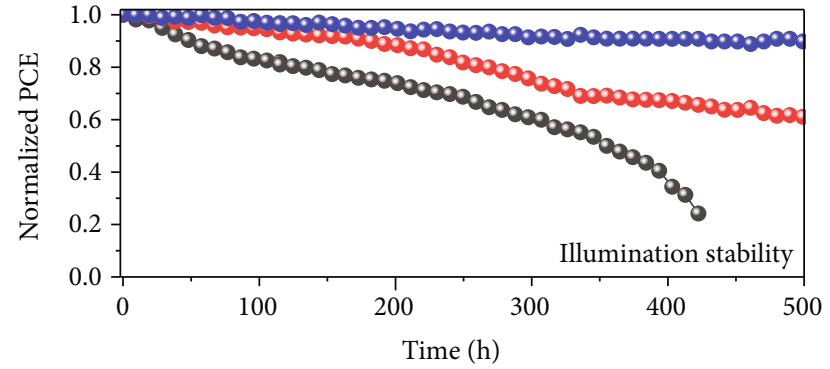

(a)

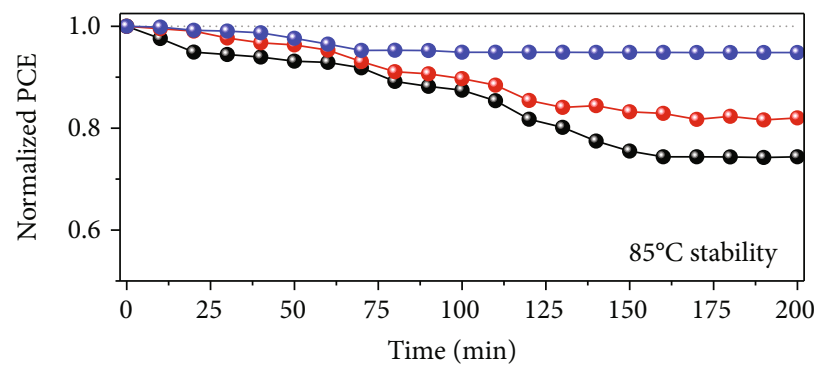

(b)

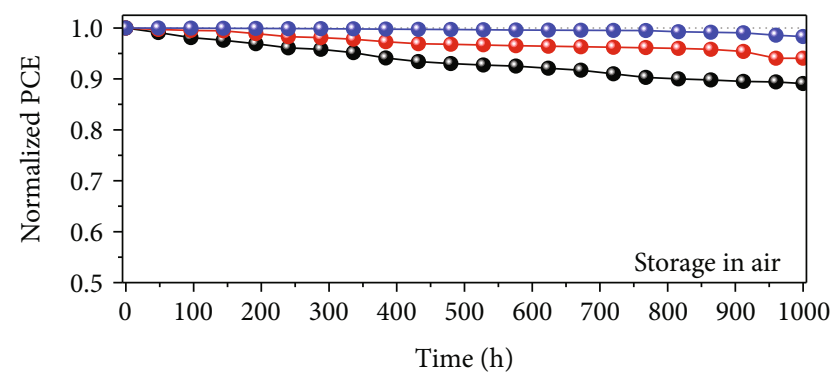

(c)

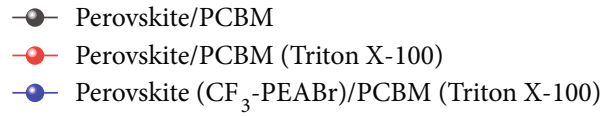

FIgURE 6: Film and device stability for different conditions. (a) Operational stability of the device with epoxy encapsulation. (b) Continuous heating at $85^{\circ} \mathrm{C}$ in ambient air. (c) Moisture stability under $\mathrm{RH} \sim 35 \%$ condition.

PEABr for perovskites. The lower trap density should be associated with the coordination between $\mathrm{CF}_{3}-\mathrm{PEABr}$ and halide dangling bonding located at the perovskite surface. All of these results are well consistent with the material characterization.

To further investigate the effect of $\mathrm{CF}_{3}-\mathrm{PEABr}$ passivation on device stability, we measured the illumination stability, $85^{\circ} \mathrm{C}$ thermal stability, and long storage stability of the devices. Firstly, we measured the light soaking stability of the perovskite film without encapsulation under 1.7 sunlight. It is obvious that the film with $\mathrm{CF}_{3}-\mathrm{PEABr}$ passivation shows much greater light stability than the control film. The passivated perovskite film still remains black in color while the control film has turned yellow after $24 \mathrm{~h}$ of illumination, as shown in Figure S17. Moreover, Figure 6(a) shows the illumination stability of the device that was measured for $500 \mathrm{~h}$ under one sunlight. The device was encapsulated by epoxy and glass. We demonstrate that the device with $\mathrm{CF}_{3}$ PEABr passivation shows better operational light stability than the conventional device. After continuous light illumination for $500 \mathrm{~h}$, the PCE of the device with $\mathrm{CF}_{3}$ PEABr passivation could retain $90 \%$ of the initial efficiency which is much higher than that of the conventional device. Fortuitously, the device with a modified PCBM (Triton X$100)$ layer also retains $60 \%$ of its initial PCE. Moreover, the thermal stability of devices with different conditions was measured under $85^{\circ} \mathrm{C}$ continuous heating, as shown in Figure 6(b). It is found that the device based on the perovskite with $\mathrm{CF}_{3}-\mathrm{PEABr}$ passivation still retains $94 \%$ of the initial PCE after $200 \mathrm{~min}$ without encapsulation, while the device without aromatic cation passivation exhibits poor stability $(74 \%$ of the initial PCE for the conventional device and $82 \%$ of the initial PCE for the device with a modified PCBM (Triton X-100) layer). In addition, we measured the long-term storage stability in ambient air with $\mathrm{RH} \sim 35 \%$ for $1000 \mathrm{~h}$, as shown in Figure 6(c). As expected, the device with $\mathrm{CF}_{3}-\mathrm{PEABr}$ passivation exhibits better PCE stability, retaining $98 \%$ of the initial PCE. Meanwhile, the device with a modified PCBM (Triton X$100)$ layer also retains $94 \%$ of the initial PCE, larger than that of the conventional device (89\%). Absolutely, the improved stability of target devices is ascribed to the lower trap-assisted states and hydrophobic surface of perovskites due to the hydrophobic nature of $\mathrm{CF}_{3}-\mathrm{PEABr}$. The hydrophobic trifluoromethyl functional group $-\mathrm{CF}_{3}-\mathrm{PEA}^{+}$ that existed at the terminal of the crystal acts as the dynamic barrier to prevent the $\mathrm{H}_{2} \mathrm{O}$ or $\mathrm{O}_{2}$ from ambient air into perovskite films in high-humidity environments.

\section{Conclusion}

In summary, we demonstrate an efficient strategy of surface passivation, wherein $\mathrm{CF}_{3}-\mathrm{PEABr}$ large organic cations with the electron-withdrawing trifluoromethyl functional group could form coordination with halide dangling bonds at the perovskite crystal surface and reduce trap-assisted states that existed in the perovskite film, leading to efficiently increased perovskite film quality and suppressed nonradiative carrier recombination. Meanwhile, a surfactant Triton X-100 is adopted to solve the hydrophobic nature properties of the $-\mathrm{CF}_{3}$ functional group and provide potential interaction between the PCBM layer and the perovskite to enhance charge transfer near the perovskite surface. As a result, the PSCs with $\mathrm{CF}_{3}-\mathrm{PEABr}$ surface passivation show significantly enhanced efficiency and better stability, wherein a higher PCE of $21.3 \%$ and a larger $V_{\text {oc }}$ of $1.15 \mathrm{~V}$ are obtained. The lower energy loss $(0.42 \mathrm{eV})$ is related to the reduced defects in perovskite films and suppressed nonradiative carrier recombination. Meanwhile, the device with $\mathrm{CF}_{3}-\mathrm{PEABr}$ surface passivation shows better light soaking, $85^{\circ} \mathrm{C}$ thermal, and long-term storage stabilities without any encapsulation after $1000 \mathrm{~h}$. Our results provide a facile way to enhance the performance and the stability of perovskite solar cells. 


\section{Data Availability}

Data used to support the findings of this study are included within the article and supplementary information file(s).

\section{Conflicts of Interest}

The authors declare no conflicts of interest.

\section{Authors' Contributions}

L. Zhou conducted the experiment. J.J. Chang conceived the idea. J. Su contributed to the first-principles calculations. X. Guo, T. Li, and J. Ma contributed to some characterizations of perovskite films. J.J. Chang, J.C. Zhang, and Y. Hao supervised the project. L. Zhou, J. Su, Z.H. Lin, and J.J. Chang wrote the paper.

\section{Acknowledgments}

This work was financially supported by the National Natural Science Foundation of China (61704131, 61804111), National Key Research and Development Program of China (Grant 2018YFB2202900), Key Research and Development Program of Shaanxi Province (Grant 2020GY-310), 111 Project (B12026), Fundamental Research Funds for the Central Universities, China Scholarship Council, and Excellent Sino-foreign youth exchange program by CAST.

\section{Supplementary Materials}

Experimental details. XRD spectra of $2 \mathrm{D}$ perovskites $\left(\mathrm{CF}_{3}\right.$ $\mathrm{PEA})_{2} \mathrm{PbI}_{2} \mathrm{Br}_{2}$. FWHM and PL perovskites. Tauc plot spectra of perovskite films. AFM images of perovskite films. FTIR spectra of $\mathrm{CF}_{3}-\mathrm{PEABr}$ powder and perovskites. XPS spectra of the perovskite films. UPS results of perovskites and band energy level scheme. KPFM images of perovskite films. Device performance for the sample with or without Triton $\mathrm{X}-100$. The schematic chemical structure of Triton X-100. The performance of the device with PEABr surface passivation. Distribution of device performance for different conditions of Triton X-100. The device performance of PSCs with different concentrations of $\mathrm{CF}_{3}-\mathrm{PEABr}$. Hysteresis behaviors of perovskite devices with different conditions. EQE curves of perovskite devices with different conditions. $\mathrm{C}-\mathrm{V}$ curves of devices with or without $\mathrm{CF}_{3}-\mathrm{PEABr}$ treatment. The illumination stability of films with or without $\mathrm{CF}_{3}$ PEABr treatment. Cross-sectional SEM image of perovskite films. Fitting decay times of perovskite films prepared with different conditions. Fitting parameters of the EIS measurements with different conditions. (Supplementary Materials)

\section{References}

[1] M. M. Lee, J. Teuscher, T. Miyasaka, T. N. Murakami, and H. J. Snaith, "Efficient hybrid solar cells based on mesosuperstructured organometal halide perovskites," Science, vol. 338, no. 6107, pp. 643-647, 2012.

[2] A. Kojima, K. Teshima, Y. Shirai, and T. Miyasaka, "Organometal halide perovskites as visible-light sensitizers for photo- voltaic cells," Journal of the American Chemical Society, vol. 131, no. 17, pp. 6050-6051, 2009.

[3] D. P. McMeekin, G. Sadoughi, W. Rehman et al., "A mixedcation lead mixed-halide perovskite absorber for tandem solar cells," Science, vol. 351, no. 6269, pp. 151-155, 2016.

[4] B. Zhang, J. Su, X. Guo et al., "NiO/perovskite heterojunction contact engineering for highly efficient and stable perovskite solar cells," Advanced Science, vol. 7, no. 11, article 1903044, 2020.

[5] J. Du, L. Feng, X. Guo et al., "Enhanced efficiency and stability of planar perovskite solar cells by introducing amino acid to $\mathrm{SnO}_{2}$ /perovskite interface," Journal of Power Sources, vol. 455, article 227974, 2020.

[6] X. Huang, J. du, X. Guo et al., "Polyelectrolyte-doped SnO2as a tunable electron transport layer for high-efficiency and stable perovskite solar cells," Solar RRL, vol. 4, no. 1, article 1900336, 2020.

[7] L. Zhou, Z. Lin, Z. Ning et al., "Highly efficient and stable planar perovskite solar cells with modulated diffusion passivation toward high power conversion efficiency and ultrahigh fill factor," Solar RRL, vol. 3, no. 11, article 1900293, 2019.

[8] X. Guo, Z. Lin, J. Ma et al., "Low temperature combustion synthesized indium oxide electron transport layer for high performance and stable perovskite solar cells," Journal of Power Sources, vol. 438, article 226981, 2019.

[9] National Renewable Energy Laboratory (NREL), "Best research cell efficiency chart," https://www.nrel.gov/pv/ assets/pdfs/best-reserch-cell-efficiencies.20190930.pdf.

[10] L. Zhou, X. Guo, Z. Lin et al., "Interface engineering of low temperature processed all-inorganic $\mathrm{CsPb}_{2} \mathrm{Br}$ perovskite solar cells toward PCE exceeding 14\%," Nano Energy, vol. 60, pp. 583-590, 2019.

[11] Z. Liu, J. Chang, Z. Lin et al., "High-performance planar perovskite solar cells using low temperature, solution-combustionbased nickel oxide hole transporting layer with efficiency exceeding 20\%," Advanced Energy Materials, vol. 8, no. 19, article 1703432, 2018.

[12] L. Zhou, J. Chang, Z. Liu et al., "Enhanced planar perovskite solar cell efficiency and stability using a perovskite/PCBM heterojunction formed in one step," Nanoscale, vol. 10, no. 6, pp. 3053-3059, 2018.

[13] F. Zhang, B. Cai, J. Song, B. Han, B. Zhang, and H. Zeng, "Efficient blue perovskite light-emitting diodes boosted by 2D/3D energy cascade channels," Advanced Functional Materials, vol. 30 , no. 27, article 2001732, 2020.

[14] Q. Jiang, Y. Zhao, X. Zhang et al., "Surface passivation of perovskite film for efficient solar cells," Nature Photonics, vol. 13, no. 7, pp. 460-466, 2019.

[15] Z. Ni, C. Bao, Y. Liu et al., "Resolving spatial and energetic distributions of trap states in metal halide perovskite solar cells," Science, vol. 367, no. 6484, pp. 1352-1358, 2020.

[16] B. Chen, P. N. Rudd, S. Yang, Y. Yuan, and J. Huang, "Imperfections and their passivation in halide perovskite solar cells," Chemical Society Reviews, vol. 48, no. 14, pp. 3842-3867, 2019.

[17] J. J. Yoo, S. Wieghold, M. C. Sponseller et al., “An interface stabilized perovskite solar cell with high stabilized efficiency and low voltage loss," Energy \& Environmental Science, vol. 12, no. 7, pp. 2192-2199, 2019.

[18] F. Gao, Y. Zhao, X. Zhang, and J. You, "Recent progresses on defect passivation toward efficient perovskite solar cells," Advanced Energy Materials, vol. 10, no. 13, article 1902650, 2020. 
[19] T. Han, S. Tan, J. Xue, L. Meng, J. Lee, and Y. Yang, "Interface and defect engineering for metal halide perovskite optoelectronic devices," Advanced Materials, vol. 31, no. 47, article 1803515, 2019.

[20] N. J. Jeon, J. H. Noh, W. S. Yang et al., "Compositional engineering of perovskite materials for high-performance solar cells," Nature, vol. 517, no. 7535, pp. 476-480, 2015.

[21] F. Zhang and K. Zhu, "Additive engineering for efficient and stable perovskite solar cells," Advanced Energy Materials, vol. 10, no. 13, article 1902579, 2020.

[22] X. Yang, X. Zhang, J. Deng et al., "Efficient green light-emitting diodes based on quasi-two-dimensional composition and phase engineered perovskite with surface passivation," Nature Communications, vol. 9, pp. 2-9, 2018.

[23] E. Aydin, M. Bastiani, and S. Wolf, "Defect and contact passivation for perovskite solar cells," Advanced Materials, vol. 31, no. 25, article 1900428, 2019.

[24] S. Akin, N. Arora, S. M. Zakeeruddin, M. Grätzel, R. H. Friend, and M. I. Dar, "New strategies for defect passivation in highefficiency perovskite solar cells," Advanced Energy Materials, vol. 10, article 1903090, 2020.

[25] N. Li, Z. Zhu, C. Chueh et al., "Mixed cation FAxPEA1$\mathrm{xPbI} 3$ with enhanced phase and ambient stability toward high-performance perovskite solar cells," Advanced Energy Materials, vol. 7, no. 1, article 1601307, 2017.

[26] P. Chen, Y. Bai, S. Wang, M. Lyu, J.-H. Yun, and L. Wang, "In situ growth of 2D perovskite capping layer for stable and efficient perovskite solar cells," Advanced Functional Materials, vol. 28, no. 17, article 1706923, 2018.

[27] X. Zhu, M. Du, J. Feng et al., "High-efficiency perovskite solar cells with imidazolium-based ionic liquid for surface passivation and charge transport," Angewandte Chemie International Edition, vol. 60, no. 8, pp. 4238-4244, 2021.

[28] C. Ran, J. Xi, W. Gao et al., "Bilateral interface engineering toward efficient 2D-3D bulk heterojunction tin halide leadfree perovskite solar cells," ACS Energy Letters, vol. 3, no. 3, pp. 713-721, 2018.

[29] Z. Wang, Q. Lin, F. P. Chmiel, N. Sakai, L. M. Herz, and H. J. Snaith, "Efficient ambient-air-stable solar cells with 2D-3D heterostructured butylammonium-caesium-formamidinium lead halide perovskites," Nature Energy, vol. 2, no. 9, 2017.

[30] X. Zheng, Y. Hou, C. Bao et al., "Managing grains and interfaces via ligand anchoring enables $22.3 \%$-efficiency inverted perovskite solar cells," Nature Energy, vol. 5, no. 2, pp. 131140, 2020.

[31] S. Yang, J. Dai, Z. Yu et al., "Tailoring passivation molecular structures for extremely small open-circuit voltage loss in perovskite solar cells," Journal of the American Chemical Society, vol. 141, no. 14, pp. 5781-5787, 2019.

[32] S. Gharibzadeh, B. Abdollahi Nejand, M. Jakoby et al., "Record open-circuit voltage wide-bandgap perovskite solar cells utilizing 2D/3D perovskite heterostructure," Advanced Energy Materials, vol. 9, no. 21, article 1803699, 2019.

[33] Q. Zhou, L. Liang, J. Hu et al., "High-performance perovskite solar cells with enhanced environmental stability based on a ( $\left.\mathrm{p}-\mathrm{FC}_{6} \mathrm{H}_{4} \mathrm{C}_{2} \mathrm{H}_{4} \mathrm{NH}_{3}\right)_{2}\left[\mathrm{PbI}_{4}\right]$ capping layer," Advanced Energy Materials, vol. 9, no. 12, article 1802595, 2019.

[34] Q. Zhou, Q. Xiong, Z. Zhang et al., "Fluoroaromatic CationAssisted planar junction perovskite solar cells with ImprovedVOCand stability: the role of fluorination position," Solar $R R L$, vol. 4 , no. 7 , article $2000107,2020$.
[35] H. Zhu, Y. Ren, L. Pan et al., "Synergistic effect of fluorinated passivator and hole transport dopant enables stable perovskite solar cells with an efficiency near 24\%," Journal of the American Chemical Society, vol. 143, no. 8, pp. 3231-3237, 2021.

[36] Z. Fang, W. Chen, Y. Shi et al., "Dual passivation of perovskite defects for light-emitting diodes with external quantum efficiency exceeding 20\%," Advanced Functional Materials, vol. 30, no. 12, article 1909754, 2020.

[37] W.-J. Yin, T. Shi, and Y. Yan, "Unusual defect physics in CH3NH3PbI3perovskite solar cell absorber," Applied Physics Letters, vol. 104, no. 6, article 063903, 2014.

[38] X. Zheng, B. Chen, J. Dai et al., "Defect passivation in hybrid perovskite solar cells using quaternary ammonium halide anions and cations," Nature Energy, vol. 2, no. 7, article 17102, 2017.

[39] L. Zhao, N. Rolston, K. M. Lee et al., "Influence of bulky organo-ammonium halide additive choice on the flexibility and efficiency of perovskite light-emitting devices," Advanced Functional Materials, vol. 28, no. 31, article 1802060, 2018.

[40] H. Wang, X. Zhang, Q. Wu et al., "Trifluoroacetate induced small-grained $\mathrm{CsPbBr}_{3}$ perovskite films result in efficient and stable light-emitting devices," Nature Communications, vol. 10, no. 1, p. 665, 2019.

[41] S. Yuan, Z. Wang, L. Xiao et al., "Optimization of lowdimensional components of quasi-2D perovskite films for deep-blue light-emitting diodes," Advanced Materials, vol. 31, no. 44, article 1904319, 2019.

[42] L. Zeng, Z. Chen, S. Qiu et al., "2D-3D heterostructure enables scalable coating of efficient low-bandgap $\mathrm{Sn}-\mathrm{Pb}$ mixed perovskite solar cells," Nano Energy, vol. 66, article 104099, 2019.

[43] J. Chang, H. Zhu, B. Li et al., "Boosting the performance of planar heterojunction perovskite solar cell by controlling the precursor purity of perovskite materials," Journal of Materials Chemistry A, vol. 4, no. 3, pp. 887-893, 2016.

[44] Z. Wang, A. K. Baranwal, M. Akmal kamarudin et al., "Delocalized molecule surface electronic modification for enhanced performance and high environmental stability of $\mathrm{CsPb}_{2} \mathrm{Br}$ perovskite solar cells," Nano Energy, vol. 66, article 104180, 2019.

[45] Y. Li, L. Meng, Y. Yang et al., "High-efficiency robust perovskite solar cells on ultrathin flexible substrates," Nature Communications, vol. 7, no. 1, p. 10214, 2016.

[46] D. Luo, L. Zhao, J. Wu et al., "Dual-source precursor approach for highly efficient inverted planar heterojunction perovskite solar cells," Advanced Materials, vol. 29, no. 19, article 1604758, 2017. 